\title{
Distributed Control for a Cost-based Droop-free Microgrid
}

\author{
Manuel Martinez Gomez \\ Dept. of Elect. Engineering \\ University of Chile \\ Santiago, Chile \\ manuel.martinez.gmz@ieee.org
}

\author{
Claudio Burgos Mellado \\ Dept. of Elect. Engineering \\ University of Nottingham \\ Nottingham, UK \\ claudio.burgosmellado1@nottingham.ac.uk
}

\author{
Roberto Cardenas Dobson \\ Dept. of Elect. Engineering \\ University of Chile \\ Santiago, Chile \\ rcd@ieee.org
}

\begin{abstract}
This paper proposes a distributed control scheme for the economic dispatch of islanded $\mathrm{AC}$ microgrids. The control goal is the economical power-sharing of distributed generators considering the marginal cost of active and reactive power. The controller is developed in a finite-time protocol over a droop-free strategy, without restoration needs for frequency. Simulations through the software PLECS are provided for validation purposes; they show adequate behaviour of the controllers under impact loads and plug \& play operation.
\end{abstract}

Index Terms-Distributed Control, Droop-Free AC Microgrid, Economic Dispatch, Finite-Time Control,

\section{INTRODUCTION}

The reactive power supply is an important ancillary service in electricity markets, and its appropriate management is an essential element for the optimal economical operation of microgrids (MGs). The optimal economic dispatch in MGs is realized by the minimization of the distributed generators' (DGs') cost functions [1], and it can include reactive power to improve the system operation. The economic function for converter-interfaced DGs comes from their power source, which can be a battery energy storage system or a conventional fuel-based generator. Some works, using centralized approaches, consider reactive power cost functions to realize the economic optimization problem [2]-[4]. However, this process involves high bandwidth communications and computation times.

The formulation of a cost function for reactive power was originated in the optimal power flow of electric power systems; the reactive power sharing is determined by the solution of an optimization problem [2], [5]. In particular, in the economic dispatch approach of the optimal power flow, the cost functions of active power, reactive power and power losses are included [6], [7]. Since there is no actual cost for the generation of reactive power, concepts like opportunity cost, power losses and cost of synchronous condensers are considered by the literature to distribute the reactive power more efficiently [3], [8], [9]. The reactive power cost function, in a deregulated electricity market, can be represented by the

This work was funded by the National Agency for Research and Development (ANID) / Scholarship Program / DOCTORADO BECAS CHILE/2019 - 21191757. Additionally, in part by the Advanced Center for Electrical and Electronic Engineering (AC3E) under Basal Project FB0008. weighted coefficients of the cost function of active power [10]. The weights come from the relation of the triangle of powers, i.e. the weights depend on the power factor $\cos (\phi)$.

In recent literature, authors have proposed a distributed cooperative control algorithms to perform the secondary control and power-sharing of AC MGs. In [11], it is proposed a consensus protocol for secondary control with a consensus protocol inside the voltage and frequency loops. The protocols are constructed based on a linear model obtained by means of the input-output feedback-linearisation technique. In [12], the authors studied further details about the inclusion of power-sharing features in the previous formulation. A slightly different approach was made by [13], where a distributedproportional-integral (DAPI) controller was elaborated. This distributed protocol uses the error in average frequency of the MG as an auxiliary variable to perform the frequency restoration. This controller also incorporates the reactive powersharing in the voltage loop, proposing a trade-off parameter for the voltage restoration weight. Another work proposed a cooperative controller replacing the frequency droop [14]. The authors drawn from the converter's phase dynamics to avoid additional integrators in the frequency restoration loop, allowing a faster secondary control response without frequency measurements. This approach also benefits from the voltage observer proposed in [15]. Following a similar path, in [16] a multi-functional controller was made by the combination of [14] and [13]. For the economic dispatch consideration, several works such as [17], [18] have adapted consensus protocols of incremental cost replacing the conventional active power.

In terms of reactive power regulation, works as [13], [16] give an equal contribution for every DG. However, this egalitarian distribution of reactive power causes a large control effort due voltage restoration and line impedances in the MG. Also, it would not provide an actual economical benefit for MGs; Especially, for MGs that can implement a cost-based reactive remuneration [10].

Motivated by the above discussion, this work proposes a distributed strategy that combines the Incremental Cost (IC) and Reactive Marginal Cost (RMC) consensus in an isolated AC MG. To the best of the authors' knowledge, secondary distributed strategies implementing such reactive cost functions for reactive power-sharing have not been proposed. 


\section{Distributed COOPERATIVE CONTROL OF AC MICROGRIDS}

A. Design of a distributed finite-time controller for incremental cost

For an $\mathrm{AC} \mathrm{MG}$ of $N$ DGs, the dynamics of the i-th converter's frequency by implementing primary and secondary control actions is represented as follows:

$$
\omega_{i}(t)=\omega^{*}-m_{i} P_{i}(t)+\delta \omega_{i}(t)
$$

where $\omega^{*}$ is the MG's reference frequency, $m_{i}[\mathrm{~V} / \mathrm{kW}]$ is the droop coefficient for primary control, and $\delta \omega_{i}[\mathrm{rad} / \mathrm{s}]$ is the secondary control deviation. Commonly, $\delta \omega_{i}(t)=\delta \omega_{i}^{1}\left(e_{i}^{P}(t)\right)+$ $\delta \omega_{i}^{2}\left(e_{i}^{\omega}(t)\right)$ where $e_{i}^{P}(t)$ and $e_{i}^{\omega}(t)$ are tracking error terms for power-sharing and frequency restoration, respectively [12], [19]. For simplicity, the droop-free strategy reported in [14], [20] is adopted, where $m_{i}=0$ causing no steady-state frequency deviations so no frequency restoration loop is required, i.e. $\delta \omega_{i}(t)=\delta \omega_{i}^{1}\left(e_{i}^{P}(t)\right)$.

Differently from [14], [20], the power variable is changed for an IC variable, so the converters' phase angle dynamics is the following:

$$
\theta_{i}(t)=\int_{0}^{t} \omega^{*} d \tau+\int_{0}^{t} e_{i}^{\lambda}(\tau) d \tau
$$

where $\delta \omega_{i}(t)=e_{i}^{\lambda}(t)$ is a compensation term for the transient disagreement of IC. In the following, the notation of variables is simplified omitting their dependency of time, i.e. $e_{i}^{\lambda}(t)=e_{i}^{\lambda}$. The error $e_{i}^{\lambda}$ is obtained from the following proposed consensus protocol

$$
\left.\delta \omega_{i}=c_{\lambda} \operatorname{sig}\left(\sum_{j \in \mathcal{N}_{i}} a_{i j}\left(\lambda_{j}-\lambda_{i}\right)\right)^{\gamma}\right\} \begin{gathered}
\text { Incremental } \\
\text { cost } \\
\text { regulator }
\end{gathered}
$$

where $\lambda$ is the IC consensus variable $\left(\lambda_{i}=2 \alpha_{i} P_{i}+\beta_{i}\right)$ with $\mathcal{N}_{i}$ the neighbourhood set for $\mathrm{i}$-th $\mathrm{DG}$, and the parameters $\gamma \in(0,1)$ and $c_{\lambda}>0$ determining the finite-time convergence [21]. (it is denoted the function $\operatorname{sig}[\cdot]^{\gamma}$ as $\operatorname{sig}[\cdot]^{\gamma}=\operatorname{sign}(\cdot)|\cdot|{ }^{\gamma}$ )

The following assumptions are considered in order to further analyze the behaviour of the proposed controller:

Assumption 1. The communication graph $\mathcal{G}$ of the $M G$ is undirected and connected, with at least one spanning tree.

Assumption 2. The converter's internal control loops are decoupled with respect the power filter measurement such that they do not interfere with the primary and secondary control dynamics.

Theorem 1. Consider the control protocol described in (3) implemented by the DGs of an AC MG. Under Assumptions 1 and 2, the DG's incremental cost synchronize in finite-time $T_{\lambda} \leq \frac{V(0)^{1-p}}{M(1-p)} \forall M>0, p \in(0,1)$ and $V(\cdot)$ is a LyapunovKrasovskii candidate function.

Proof. The disagreement vector of IC is constructed as $\delta^{\lambda}=$ $\left(\delta_{1}^{\lambda}, \delta_{2}^{\lambda}, \ldots, \delta_{N}^{\lambda}\right)$ with $\delta_{i}^{\lambda}=\int e_{i}^{\lambda}=\lambda_{i}-\frac{1}{N} \sum_{j=1}^{N} \lambda_{j}$, and its derivative as $\dot{\delta}_{i}^{\lambda}=c_{\lambda} \sum_{j=1}^{N} a_{i j} \operatorname{sig}\left[\delta_{j}^{\lambda}-\delta_{i}^{\lambda}\right]^{\gamma}$.
Let the Lyapunov candidate be given by

$$
V=\frac{1}{2} \delta^{\lambda}\left(\delta^{\lambda}\right)^{T}
$$

Differentiating and using (Lemma 2 [22]), one has

$$
\begin{aligned}
& \dot{V}=\left(\delta^{\lambda}\right)^{T} \dot{\delta}^{\lambda}=\sum_{i, j=1}^{N} c_{\lambda} \delta_{i}^{\lambda} a_{i j} \operatorname{sig}\left[\delta_{j}^{\lambda}-\delta_{i}^{\lambda}\right]^{\gamma} \\
& \dot{V} \leq-\frac{1}{2}\left(\sum_{i, j=1}^{N}\left(c_{\lambda} a_{i j}\right)^{\frac{2}{1+\gamma}}\left(\delta_{j}^{\lambda}-\delta_{i}^{\lambda}\right)^{2}\right)^{\frac{1+\gamma}{2}}
\end{aligned}
$$

By defining $\left[a_{i j}^{\lambda}\right]=\left[\left(c_{\lambda} a_{i j}\right)^{\frac{2}{(1+\gamma)}}\right]$, and $L^{\lambda}\left(a_{i j}^{\lambda}\right)$ as the Laplacian matrix of the graph, eq. (6) results in

$$
\dot{V} \leq-\frac{1}{2}\left(\left(\delta^{\lambda}\right)^{T} L^{\lambda} \delta^{\lambda}\right)^{\frac{1+\gamma}{2}}
$$

From (Lemma 3 [22]), one has the following relation

$$
2\left(\delta^{\lambda}\right)^{T} L^{\lambda} \delta^{\lambda} \geq 2 \eta_{2}\left(L^{\lambda}\right)\left(\delta^{\lambda}\right)^{T} \delta^{\lambda}>0
$$

with $\eta_{2}\left(L^{\lambda}\right)$ as the second eigenvalue of $L^{\lambda}$. Replacing (4) and (8) into (7) gives

$$
\begin{aligned}
\dot{V} & \leq-\frac{1}{2}\left(4 \eta_{2}\left(L^{\lambda}\right) V\right)^{\frac{1+\gamma}{2}} \\
& \leq-2^{\gamma} \eta_{2}\left(L^{\lambda}\right)^{\frac{1+\gamma}{2}} V^{\frac{1+\gamma}{2}} \\
& \leq-M V^{p}
\end{aligned}
$$

where $M=2^{\gamma} \eta_{2}\left(L^{\lambda}\right)^{\frac{1+\gamma}{2}}$ and $p=\frac{1+\gamma}{2}$ are positive constants as long as $c_{\lambda}>0$ and $\gamma \in(0,1)$. Therefore, (9) satisfies (Lemma 1, [22]) and completes the proof.

B. Design of a distributed finite-time controller for reactive marginal cost

Based on the voltage control loop of [14], the following voltage dynamics are proposed:

$$
E_{d i}=E^{*}-n Q_{i}+\delta E_{i}^{1}+\delta E_{i}^{2}
$$

where $E_{d i}$ is the local voltage in the d-q reference frame and $E^{*}$ is the MG reference voltage. The term $n Q_{i}$ is the decentralised droop compensation. The terms $\delta E_{i}^{1}$ and $\delta E_{i}^{2}$ are control actions from PI controllers. The term $\delta E_{i}^{1}$ is obtained from a finite-time consensus, using a distributed observer for average voltage. The term $\delta E_{i}^{2}$ comes from a finite-time consensus but of new variable, the RMC. The complete proposed controller is summarized as

$$
\begin{aligned}
& \text { Average voltage regulator } \\
& \qquad \begin{array}{l}
\delta E_{i}^{1}=k_{p}^{E}\left(u_{i}^{E}\right)+k_{i}^{E} \int_{0}^{t}\left(u_{i}^{E}\right) d \tau \\
u_{i}^{E}=g_{i} \operatorname{sig}\left[E^{*}-\bar{E}_{i}\right]^{\Omega} \\
\bar{E}_{i}=E_{d i}+\int_{0}^{t}\left(\sum_{j \in \mathcal{N}_{i}} a_{i j} \operatorname{sig}\left[\bar{E}_{j}-\bar{E}_{i}\right]^{\alpha}\right) d \tau
\end{array}
\end{aligned}
$$


Reactive cost regulator

$$
\begin{aligned}
\delta E_{i}^{2} & =k_{p}^{\Psi}\left(u_{i}^{\Psi}\right)+k_{i}^{\Psi} \int_{0}^{t}\left(u_{i}^{\Psi}\right) d \tau \\
u_{i}^{\Psi} & =c_{\Psi} \operatorname{sig}\left[\sum_{j \in \mathcal{N}_{i}} a_{i j}\left(\Psi_{j}-\Psi_{i}\right)\right]^{\beta}
\end{aligned}
$$

where $\bar{E}_{i}$ and $\Psi_{i}$ are the average voltage and RMC estimations of the i-th DG, $\left\{k_{p}^{E}, k_{i}^{E}, k_{p}^{\Psi}, k_{i}^{\Psi}\right\}>0$ are PI control parameters, $\{\alpha, \beta, \Omega\} \in(0,1)$ and $c_{\Psi}>0$ are convergence speed parameters. The pinning gain $g_{i}$ indicates the access of the DG to the reference value $E^{*}$. The variable $\Psi_{i}$ comes from a reactive cost function based on [10], and it incorporates the available apparent power for avoiding the DGs overloading. Therefore, $\Psi$ is given by:

$$
\Psi_{i}=2 \alpha_{i}^{\prime} S_{\text {disp i }} Q_{i}+\beta_{i}^{\prime}, \quad \text { with } \quad S_{\text {disp i }}=\frac{S_{\operatorname{max~i}}-S_{i}}{S_{\operatorname{max~i}}}
$$

where $S_{i}$ and $Q_{i}$ are the local measured apparent and reactive powers. Coefficients $\alpha_{i}^{\prime}=\alpha_{i} \sin ^{2}(\phi)$ and $\beta_{i}^{\prime}=\beta_{i} \sin (\phi)$ are weighted versions of the DG's cost function parameters (used for IC) with $\phi$ as the power factor's angle.

Theorem 2. Let Assumptions 1 and 2 hold. Consider the $i$-th $D G$ controlled with the regulators (11) and (12). Then, given a desired reference $E^{*}$, the DG voltages $E_{i}(t)$ of the $M G$ will converge near reference while achieving an optimal reactive power-sharing within a finite time $T_{E}$ for all $t \geq T_{E}$.

Proof. The demonstration is similar to the Theorem 1. The disagreement vectors are $\delta^{E}=\left(\delta_{1}^{E}, \ldots \delta_{N}^{E}\right)$ and $\delta^{\Psi}=$ $\left(\delta_{1}^{\Psi}, \ldots \delta_{N}^{\Psi}\right)$, with $\delta_{i}^{E}=\bar{E}_{i}-E^{*}$ and $\delta_{i}^{\Psi}=\Psi_{i}-$ $\frac{1}{N} \sum_{j=1}^{N} \Psi_{j}$. The derivatives are $\dot{\delta}_{i}^{E}=g_{i} \operatorname{sig}\left[\delta^{E}\right]^{\Omega}$ and $\dot{\delta}_{i}^{\Psi}=$ $c_{\Psi} \sum_{j=1}^{N} a_{i j} \operatorname{sig}\left[\delta_{j}^{\Psi}-\delta_{i}^{\Psi}\right]^{\beta}$. The Lyapunov function can be constructed as

$$
V=V_{E}+V_{\Psi}=\frac{1}{2}\left(\delta^{E}\right)\left(\delta^{E}\right)^{T}+\frac{1}{2}\left(\delta^{\Psi}\right)\left(\delta^{\Psi}\right)^{T}
$$

Following the steps of Theorem 1, it is had

$$
\dot{V} \leq-M_{1} V_{E}^{\frac{1+\Omega}{2}}-M_{2} V_{\Psi}^{\frac{1+\beta}{2}}=-\kappa V^{p}
$$

where $M_{1}=2^{\Omega} \eta_{2}(G)^{\frac{1+\Omega}{2}}, M_{2}=2^{\beta} \eta_{2}\left(L^{\Psi}\right)^{\frac{1+\beta}{2}}$, and coefficients $p$ and $\kappa$ are positive if and only if $c_{\Psi}>0$ and $\{\Omega, \beta\} \in(0,1)$ (also $\Omega=\beta$ ). Therefore, (15) satisfies (Lemma 1, [22]), i.e. $V(t)$ reaches zero at finite time $T_{E} \leq \frac{V(0)^{1-p}}{\kappa(1-p)}$.

\section{CASE STUDIES}

In this section, it is described the evaluation process for the proposed controllers. Firstly, an AC MG is modelled in software PLECS®. The modelled $\mathrm{MG}$ is based on a radial distribution system of 5 DGs, 3 loads and 3 buses. Fig. 1 summarizes the elements considered in the control loop of each DG; A packaging method transmits the control variables with a rate of $100[\mathrm{~Hz}]$ (10 [ms] delay). The communication topology is redundant for reliability purposes, then each DG communicates with its nearest neighbours following the structure of the distribution system. The parameters of the MG are shown in Table I, and the control parameters are shown in Tables II-III.

\begin{tabular}{cc|cc}
\hline \hline Parameter & Value & Parameter & Value \\
\hline \hline Nom. Voltage & $220\left[\mathrm{~V}_{1 \Phi}\right]$ & Line Resist. 1-2 & $0.15[\Omega]$ \\
Load Resist. Z1 & $30.30[\Omega]$ & Line Resist. 2-3 & $0.20[\Omega]$ \\
Load Resist. Z2 & $30.00[\Omega]$ & Line Induct. 1-2 & $0.30[\mathrm{mH}]$ \\
Load Resist. Z3 & $10.1+j 0.78[\Omega]$ & Line Induct. 2-3 & $0.25[\mathrm{mH}]$ \\
\hline
\end{tabular}

TABLE I: Distribution system for AC MG.

\begin{tabular}{cc|cc|cc}
\hline \hline Parameter & Value & Parameter & Value & Parameter & Value \\
\hline \hline$\omega_{c}$ & 18.84 & $k_{i}^{V}$ & 14.01 & $c_{\lambda}$ & 0.03 \\
$m$ & 0.00 & $k_{p}^{E}$ & 5.00 & $\alpha$ & 0.50 \\
$n$ & $2.6 \mathrm{e}-3$ & $k_{i}^{E}$ & 47.12 & $\beta$ & 0.50 \\
$R_{d}$ & 0.85 & $k_{p}^{\Psi}$ & 0.05 & $\gamma$ & 0.50 \\
$R_{v}$ & 3.40 & $k_{i}^{\Psi}$ & 1.57 & $\Omega$ & 1.00 \\
$k_{p}^{I}$ & 4.26 & $c_{\Psi}$ & 2200 & & \\
$k_{i}^{I}$ & 8389 & $k_{p}^{\lambda}$ & 0.05 & & \\
$k_{p}^{V}$ & 0.05 & $k_{i}^{\lambda}$ & 0.94 & & \\
\hline * The parameters $R_{d}, R_{v}, k_{p}^{I}, k_{i}^{I}, k_{p}^{V}$ and $k_{i}^{V}$ account for the direct \\
and quadrature axis controllers in a rotating reference frame strategy.
\end{tabular}

TABLE II: Control parameters of AC MG's DGs.

\begin{tabular}{cc|cc|cc}
\hline \hline Parameter & Value & Parameter & Value & Parameter & Value \\
\hline \hline$\omega_{c}$ & 62.83 & $k_{\Omega}$ & 0.3 & $k_{Q}$ & 0.3 \\
$m$ & $1.9 \mathrm{e}-4$ & $k_{E}$ & $7 \mathrm{e}-3$ & $\beta$ & $6 \mathrm{e}-3$ \\
$n$ & $1.9 \mathrm{e}-2$ & $k_{\lambda}$ & 2.0 & & \\
\hline
\end{tabular}

* The rest of the parameters are the same as Table II

TABLE III: Control parameters of AC MG's DGs used for DAPI comparison.

There are three groups of studies for simulations. A first analysis is realized concerning a comparison between the proposed control algorithms, i.e. the finite-time protocol of (11)-(12) as well as its linear variation $(\{\alpha, \beta, \gamma\}=1)$, and the reported strategy in [18]. The controller in [18] is selected because it uses a simple and validated structure, initially proposed by [13]. For the implementation, it is changed the original control variable $Q_{i}^{p . u}$ to $\Psi_{i}$. It is also considered only one DG to realize the comparisons.

A second analysis is carried out using the simulation of the proposed finite-time protocol in (11)-(12). It is analyzed how the DGs behaves in different operation conditions.

A third analysis consists of comparing the performance of the distributed secondary controllers when using either the proposed RMC power-sharing or the conventional reactive power-sharing, i.e. $\left(Q_{i}^{p . u}=Q_{j}^{p . u} \forall i, j \in \mathcal{N}\right)$. For simplicity, it is shown the comparison using only the asymptotic version of the controllers in (3), (11) and (12) $(\{\alpha, \beta, \gamma, \Omega\}=1)$

For the above analyses, there are two operation cases simulated, they are Load Changing and Plug \& Play operations. 

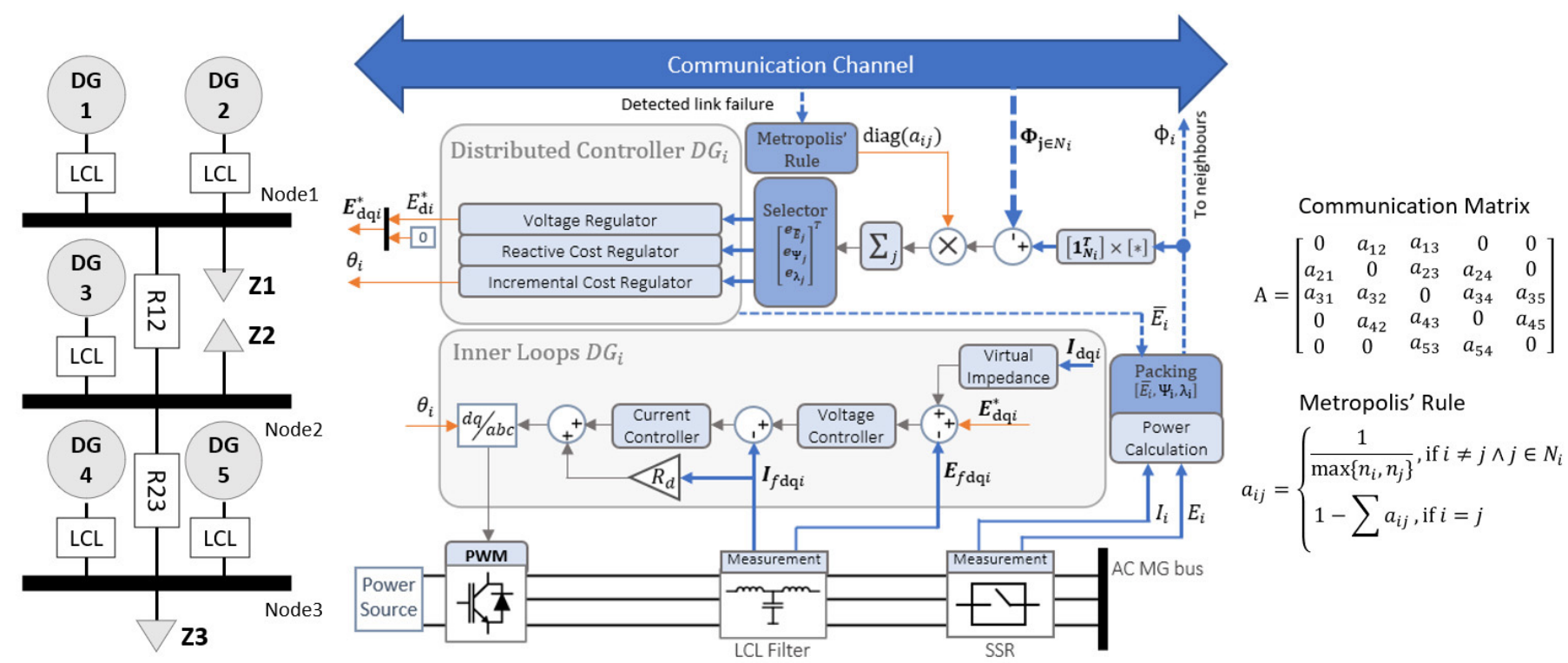

Fig. 1: Control scheme over the i-th DG in AC MG

Case 1: Load changing operation. Here a controlled load impact is made, in such a way that the MG presents a noticeable change in the power supplied by the units, and thus to be able to study the performance of the controller in transient states. The amount of the load impact varies depending on the test and simulation model.

Case 2: Plug \& Play operation. The behaviour of the MG against the disconnection and re-connection of DGs is studied. In particular, the failure of only one unit at a time will be considered. This involves DG3 unit failing and disconnecting from the network; Then, the communications matrix is updated to avoid instability of the system. The failure contemplates that the local controller loses all the values stored in the memory of its integrators. For unit re-connection, the voltage measurement after the contractor is considered to refer to the internal voltage loop. Also, it is included a synchronization stage through a PLL which is in charge of synchronizing the voltage generated before the PCC.

\section{REsults}

\section{A. Comparison with benchmark strategy}

1) Case 1: Load changing operation: The results in Fig. 2 suggest that the finite-time algorithm improves the convergence when compared with the linear consensus. Respecting the DAPI, it shows a similar convergence than the linear version of the proposed protocol, excepting for the voltage loop. This is because DAPI deals with a trade-off between reactive power sharing and voltage restoration, therefore, a reduction in voltage settling-time deteriorates the reactive power-sharing between DGs. This issue is partially solved by the use of voltage observer in the proposed controller.

2) Case 2: Plug and Play operation: The graphs of the Fig. 3 show an over-effort on the part of the DAPI controller to overcome the re-connection of a unit. It is important to note that the re-connection takes part with the secondary controller activated in these tests. In addition, the incoming DG does not know the IC, since in the disconnection a reset of its values is carried out in order to simulate realistic conditions. Although they can be developed more elaborate communication protocols that acquire the IC measurement in advance to reduce the transient, this test demonstrates the inability of the DAPI controller to overcome by its own this difficulty, unlike the other strategies proposed in this thesis. Moreover to the above, figures a) and c) again show, as in the case of load impact, a slow transient response from $t=25[s]$ where the DG3 unit is disconnected and the other DGs experiments a virtual load impact.

Regarding the inclusion of the finite-time protocol, its contribution is noted again. From Fig. 3, it is possible to see that the transients of the finite-time protocol are smoother, as seen in the case of the load impact. This result allows validating previous results obtained in other works such as [22], where it is shown that the inclusion of finite-time algorithms in the feedback benefits the convergence time.

\section{B. Performance of proposed controller}

1) Case 1: Load changing operation: Fig. 4 shows the results with load impacts. At $\mathrm{t}=15$ [s] a load impact occurs at $\mathrm{Z3}$, then at $\mathrm{t}=25$ [s] an opposite load impact occurs, recovering the initial condition. The curves depicts a fast cooperative control action to redistribute the power before changes in demand. The settling time for the controls are around the second, with the voltage control being the slowest of them. In the cases of frequency and average voltage, both return to their nominal values after transients. Similarly, the variables of IC and RMC are synchronized between DGs in the steady-state, achieving new consensus values after the load variations. 

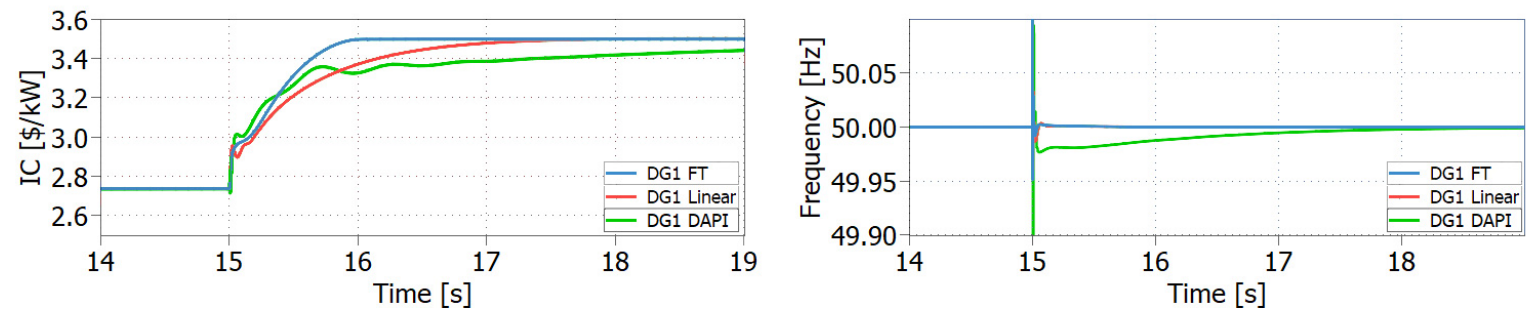

Fig. 2: Simulation comparison of DG1 for the consensus protocols in [18] and (11)-(12) (including its asymptotic version) under a load impact
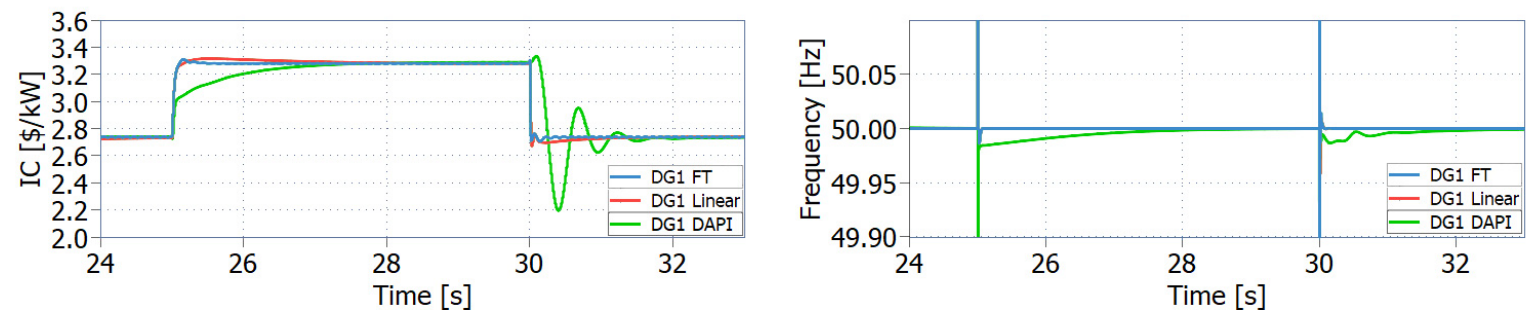

Fig. 3: Simulation comparison of DG1 for the consensus protocols in [18] and (11)-(12) (including its asymptotic version) under a plug \& play operation

2) Case 2: Plug and Play operation: Fig. 5 shows the results of the plug \& play. At $\mathrm{t}=35$ [s] the disconnection of DG3 occurs, then at $\mathrm{t}=45[\mathrm{~s}]$ the unit reconnects. The curves show adequate behaviour of the control strategy. The remaining units are capable of supplying the necessary power and the contributions of active and reactive power are redistributed according to the cost parameters of each DG. As in the case of load impact, transients recover quickly within one second.

\section{Comparison between reactive power control goals}

1) Case 1: Load changing operation: From Fig. 6, it can be observed that under load impact the control effort produced by the conventional power-sharing strategy produces an increased transient oscillation in both active and reactive powers, conversely to the proposed strategy of RMC. Therefore, it is suggested that the conventional reactive power-sharing reduces the feasible control bandwidth of the IC consensus when compare with an RMC power-sharing strategy.

2) Case 2: Plug and Play operation: The behaviour described for the load impact case can be also noticed in the plug \& play operation, Fig.7, where the re-connection of the DG3 in the MG produces a sudden power variation that is greater in magnitude and oscillating when the conventional reactive power-sharing is used. It is worth noting that the latter issue can be avoided delaying the activation of secondary control of the re-connecting unit or using virtual impedance compensation techniques reported in the literature, like the soft-starter shown in [19].

\section{CONCLUSiOns}

From simulations, it can be seen that the proposed strategy is capable of reaching the consensus of IC and RMC simul- taneously, without producing oscillations or negative effects on the secondary control loop. These results give rise for the incorporation of new variables, as operating cost components and opportunity costs related to the supply of reactive power, in the distributed optimal dispatch; In particular, it benefits the reactive power-sharing based on cooperative control of DGs within an isolated AC MG, which does not perform an actual optimization problem in hardware.

\section{ACKNOWLEDGEMENT}

The authors thank the Department of Electrical Engineering (DIE) of the University of Chile for the given support.

\section{REFERENCES}

[1] D. E. Olivares et al., "Trends in microgrid control," IEEE Trans. Smart Grid, vol. 5, no. 4, pp. 1905-1919, 2014.

[2] M. Baughman and S. Siddiqi, "Real-time pricing of reactive power: theory and case study results," IEEE Trans. Power Syst., vol. 6, no. 1, pp. 23-29, 1991.

[3] S. Hao and A. Papalexopoulos, "Reactive power pricing and management," IEEE Power Engineering Review, vol. 17, no. 2, p. 49, 1997.

[4] J. Lamont and J. Fu, "Cost analysis of reactive power support," IEEE Trans. Power Syst., vol. 14, no. 3, pp. 890-898, 1999.

[5] S. Granville, "Optimal reactive dispatch through interior point methods," IEEE Trans. Power Syst., vol. 9, no. 1, pp. 136-146, 1994.

[6] I. Khan, Y. Xu, H. Sun, and V. Bhattacharjee, "Distributed Optimal Reactive Power Control of Power Systems," IEEE Access, vol. 6, pp. 7100-7111, 2017.

[7] B. R. Serrano and A. Vargas, "Active - Reactive power economic dispatch of very short term in competitive electric markets," IEEE Porto Power Tech Proceedings, pp. 502-507, 2001.

[8] S. Hasanpour, R. Ghazi, and M. H. Javidi, "A new approach for cost allocation and reactive power pricing in a deregulated environment," Electrical Engineering, vol. 91, no. 1, pp. 27-34, 2009.

[9] A. Kumar, "Reactive Power and FACTS Cost Models' Impact on Nodal Pricing in Hybrid Electricity Markets," Smart Grid and Renewable Energy, vol. 2, pp. 230-244, 2011. 

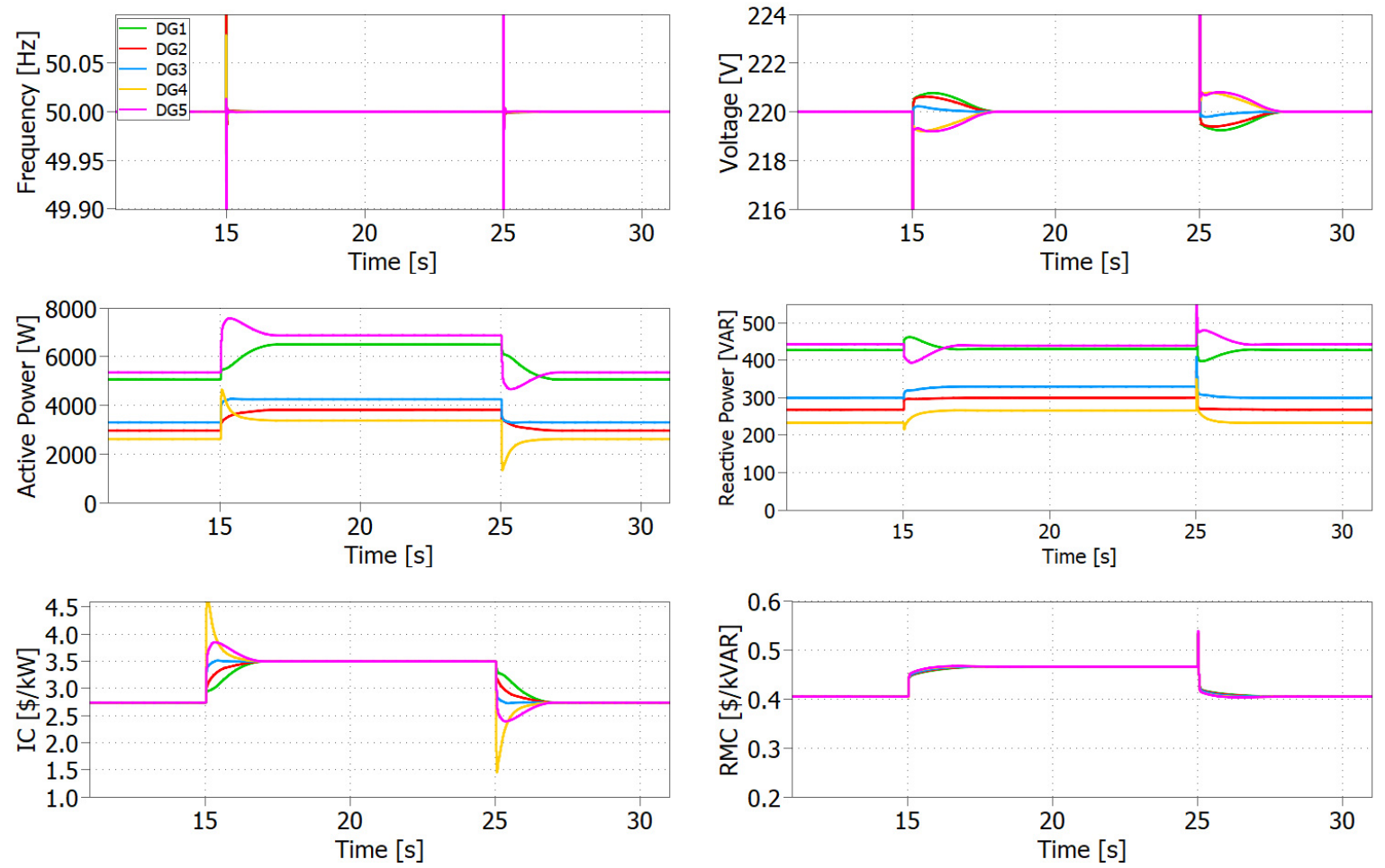

Fig. 4: Curves of simulated AC MG under load impact
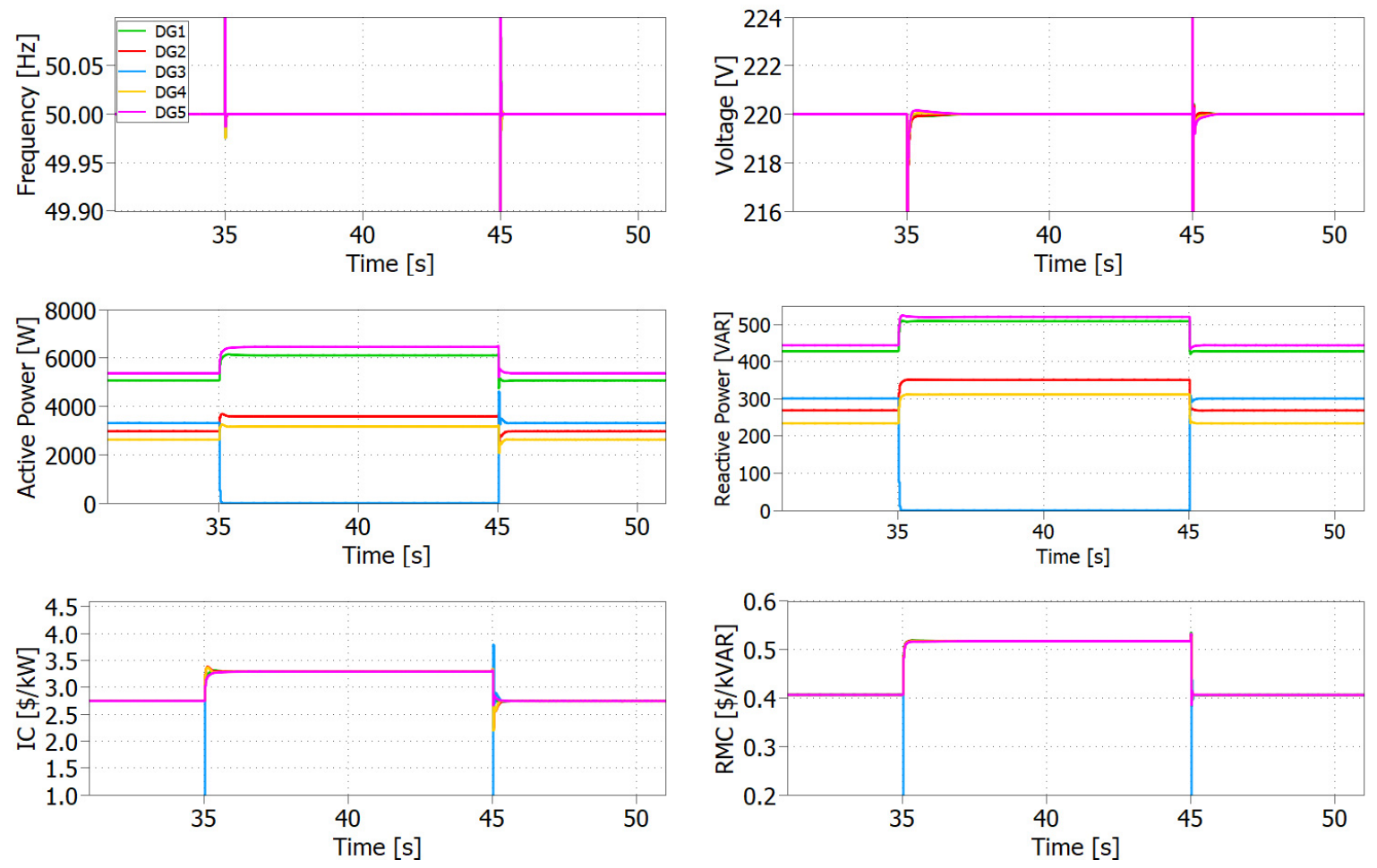

Fig. 5: Curves of simulated AC MG under plug \& play operation

[10] Y. Zhao and M. R. Irving, "A reactive power service cost allocation and pricing method in the New Deregulated electricity market environment," Proceedings of the IEEE Power Engineering Society Transmission and
Distribution Conference, pp. 1035-1040, 2006.

[11] A. Bidram, A. Davoudi, F. L. Lewis, and J. M. Guerrero, "Distributed cooperative secondary control of microgrids using feedback lineariza- 

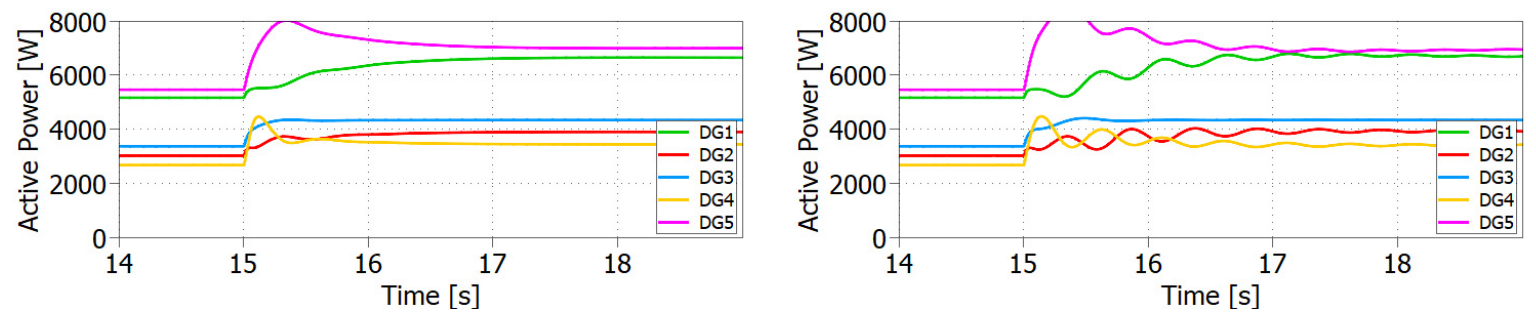

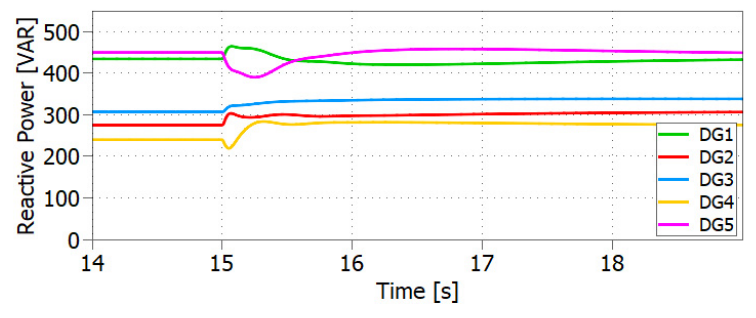

(a) Proposed RMC power-sharing

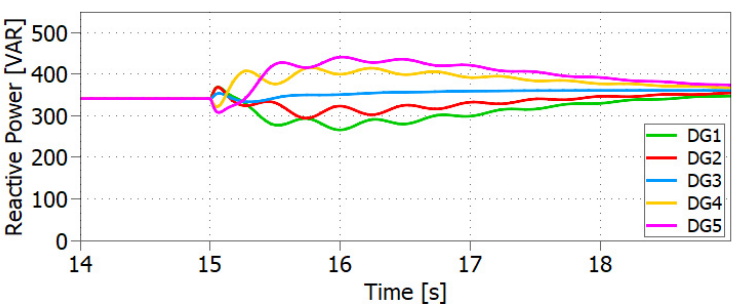

(b) Equal reactive power-sharing

Fig. 6: Simulation comparison between reactive power control goals under a load impact
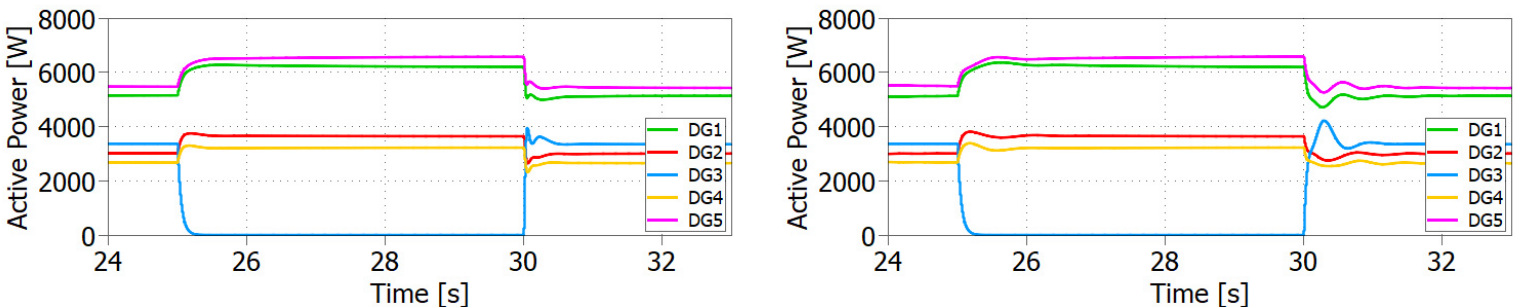

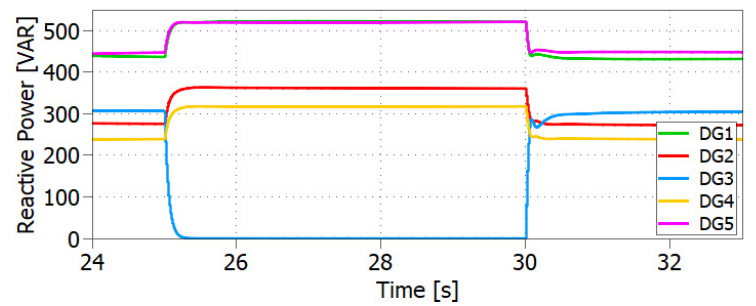

(a) Proposed RMC power-sharing

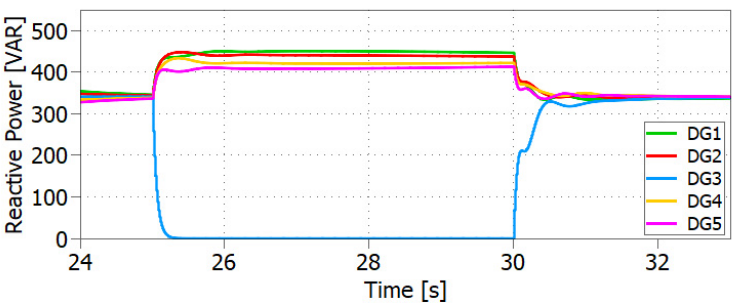

(b) Equal reactive power-sharing

Fig. 7: Simulation comparison between reactive power control goals under plug \& play operation

tion," IEEE Trans. Power Syst., vol. 28, no. 3, pp. 3462-3470, 2013.

[12] A. Bidram, A. Davoudi, F. L. Lewis, and Z. Qu, "Secondary control of microgrids based on distributed cooperative control of multi-agent systems," IET Generation, Transmission \& Distribution, vol. 7, no. 8, pp. 822-831, 2013. [Online]. Available: http://digitallibrary.theiet.org/content/journals/10.1049/iet-gtd.2012.0576

[13] J. W. Simpson-Porco, Q. Shafiee, F. Dörfler, J. C. Vasquez, J. M. Guerrero, and F. Bullo, "Secondary frequency and voltage control of islanded microgrids via distributed averaging," IEEE Trans. Ind. Electron., vol. 62, no. 11, pp. 7025-7038, 2015.

[14] V. Nasirian, Q. Shafiee, J. M. Guerrero, F. L. Lewis, and A. Davoudi, "Droop-free distributed control for ac microgrids," IEEE Trans. Power Electron., vol. 31, no. 2, pp. 1600-1617, 2016.

[15] V. Nasirian, A. Davoudi, F. L. Lewis, and J. M. Guerrero, "Distributed Adaptive Droop Control for DC Distribution Systems," IEEE Trans. Energy Convers., vol. 29, no. 4, pp. 944-956, 2014.

[16] Q. Shafiee, V. Nasirian, J. C. Vasquez, J. M. Guerrero, and A. Davoudi, "A Multi-Functional Fully Distributed Control Framework for AC Microgrids," IEEE Transactions on Smart Grid, vol. 9, no. 4, pp. 3247$3258,2018$.
[17] G. Chen, F. L. Lewis, E. N. Feng, and Y. Song, "Distributed Optimal Active Power Control of Multiple Generation Systems," IEEE Transactions on Industrial Electronics, vol. 62, no. 11, pp. 7079-7090, 2015.

[18] J. Llanos, J. Gomez, D. Saez, D. Olivares, and J. Simpson-Porco, "Economic dispatch by secondary distributed control in microgrid," in 21st European Conference on Power Electronics and Applications, 2019.

[19] J. M. Guerrero, J. C. Vasquez, J. Matas, L. G. De Vicuña, and M. Castilla, "Hierarchical control of droop-controlled AC and DC microgrids - A general approach toward standardization," IEEE Trans. Ind. Electron., vol. 58, no. 1, pp. 158-172, 2011.

[20] J. M. Rey, P. P. Vergara, M. Castilla, A. Camacho, M. Velasco, and P. Martí, "Droop-free hierarchical control strategy for inverter-based AC microgrids," IET Power Electronics, vol. 13, no. 7, pp. 1403-1415, 2020.

[21] L. Wang and F. Xiao, "Finite-time consensus problems for networks of dynamic agents," IEEE Trans. Autom. Control, vol. 55, no. 4, pp. 950-955, 2010.

[22] S. Zuo, A. Davoudi, Y. Song, and F. L. Lewis, "Distributed Finite-Time Voltage and Frequency Restoration in Islanded AC Microgrids," IEEE Trans. Ind. Electron., vol. 63, no. 10, pp. 5988-5997, 2016. 